\title{
CERTAIN ELEMENTS IN QUOTIENTS OF MEASURE ALGEBRAS
}

\author{
SADAHIRO SAEKI
}

\begin{abstract}
Let $G$ be a locally compact group, and $M(G)$ the convolution algebra with unit $\delta$ of all bounded Radon measures on $G$. Let $I$ be a left ideal in $M(G)$ and let $C \cap I^{\perp}$ be the space of all bounded continuous functions $P$ on $G$ with $\int_{G} P d \mu=0$ for all $\mu$ in I. Suppose that there exists a function $P_{0}$ in $C \cap I \perp$ such that $\left\|P_{0}\right\|_{\infty}>\lim \sup _{x \rightarrow \infty}\left|P_{0}(x)\right|$. Let also $\lambda$ and $\mu$ be two measures in $M(G)$ such that $\|\lambda+I\|=\|\mu+I\|=1$, and $(\lambda+I) * \mu \subset \delta+1$. In this paper we prove under these conditions that there exist a complex number $c$ of modulus one and a point $x_{0}$ in $G$ such that $\int_{G} P d \mu=$ $c P\left(x_{0}\right)$ for all functions $P$ in $C \cap I \perp$ with $\|P\|_{\infty} \leqq\left|\int_{G} P d \mu\right|=1$. This generalizes a theorem of $\mathrm{K}$. deLeeuw and $\mathrm{Y}$. Katznelson.
\end{abstract}

The purpose of this paper is to generalize two results due to $\mathrm{K}$. deLeeuw and Y. Katznelson [1].

Let $G$ be a locally compact (Hausdorff) space, and $C(G)$ the Banach space of bounded continuous functions on $G$. Let also $C_{0}(G)$ be the closed linear subspace of $C(G)$ consisting of all continuous functions vanishing at infinity, so that the conjugate space of $C_{0}(G)$ is $M(G)$, the space of bounded Radon measures on $G$ [2]. Writing

$$
\langle P, \mu\rangle=\int_{G} P d \mu \quad(P \in C(G), \mu \in M(G)),
$$

we identify $C(G)$ with a subspace of $M^{\prime}(G)$, the conjugate space of $M(G)$.

We fix any linear subspace $I$ of $M(G)$, and define

$$
\|\mu+I\|=\inf \{\|\nu\|: \nu \in \mu+I\} \quad(\nu \in M(G)) .
$$

We also use the following two notations:

where $0<\eta<1$.

$$
\begin{aligned}
C \cap I^{\perp} & =\{P \in C(G):\langle P, \lambda\rangle=0(\lambda \in I)\} ; \\
N_{\eta}(I) & =\left\{P \in C \cap I^{\perp}:\|P\|_{\infty} \leqq 1, \limsup _{x \rightarrow \infty}|P(x)|<1-\eta\right\},
\end{aligned}
$$

Received by the editors May 4, 1972.

AMS (MOS) subject classifications (1970). Primary 43A05; Secondary 43A10.

Key words and phrases. Locally compact group, measure, ideal.

(c) American Mathematical Society 1973 
Proposition (cf. [1, Proposition 4.1]). Let $\mu$ be any measure in $M(G)$, and suppose that

$$
\|\mu+I\|-\sup \left\{|\langle P, \mu\rangle|: P \in N_{\eta}(I)\right\}=o(\eta) \quad \text { as } \eta \rightarrow 0 ;
$$

then there exists a measure $v$ in $M(G)$ such that

(i) $\|\nu\|=\|\mu+I\|$, and

(ii) $\langle P, v\rangle=\langle P, \mu\rangle$

for all functions $P$ in $C \cap I^{\perp}$.

Proof. There exists a sequence $\left(v_{n}\right)$ of measures in $\mu+I$ such that $\lim _{n}\left\|v_{n}\right\|=\|\mu+I\|$. For a given $\varepsilon>0$, we can take a function $P_{\varepsilon}$ in $N_{\eta}(I)$ for some $0<\eta<1$ such that

$$
\|\mu+I\|-\left|\left\langle P_{\varepsilon}, \mu\right\rangle\right|<\varepsilon \eta .
$$

Let $K_{\varepsilon}$ be a compact subset of $G$ such that

Then we have

$$
\left|P_{\varepsilon}(x)\right|<1-\eta \quad\left(x \in K_{\varepsilon}^{c}\right) .
$$

$$
\begin{aligned}
\left|\left\langle P_{\varepsilon}, \mu\right\rangle\right| & =\lim _{n}\left|\left\langle P_{\varepsilon}, v_{n}\right\rangle\right| \\
& \leqq \liminf _{n}\left(\int_{K_{\varepsilon}}+\int_{K_{\varepsilon}^{c}}\right)\left|P_{\varepsilon}\right| d\left|v_{n}\right| \\
& \leqq \liminf _{n}\left\{\left|v_{n}\right|\left(K_{\varepsilon}\right)+(1-\eta)\left|v_{n}\right|\left(K_{\varepsilon}^{c}\right)\right\} \\
& =\|\mu+I\|-\eta \lim \sup \left|v_{n}\right|\left(K_{\varepsilon}^{c}\right),
\end{aligned}
$$

which, combined with (1), yields

$$
\limsup _{n}\left|v_{n}\right|\left(K_{\varepsilon}^{c}\right) \leqq \varepsilon .
$$

Since $\varepsilon>0$ was arbitrary, this assures that there exists a subnet $\left(v_{\alpha}\right)$ of $\left(v_{n}\right)$ converging to some measure $v \in M(G)$ in the weak topology $\sigma(M(G), C(G))$. In particular, we have

$$
\langle P, \mu\rangle=\lim _{\alpha}\left\langle P, v_{\alpha}\right\rangle=\langle P, v\rangle
$$

for all $P$ in $C \cap I^{\perp}$, which proves (ii). It remains to show (i). The inequality $\|\nu\| \leqq\|\mu+I\|$ is trivial, and the converse inequality follows from (*) and (ii). This completes the proof.

We now suppose that $G$ is a locally compact group, so that $M(G)$ is a Banach algebra with unit $\delta$ under convolution. The following is a generalization of the author's lemma [4, Lemma 4.2], which is originally due to K. deLeeuw and Y. Katznelson [1, Theorem 1.5] (see also [3]). 
TheOREM. Suppose that I is a left ideal in $M(G)$, and that there exists a function $P_{0}$ in $C \cap I^{\perp}$ such that

$$
\left\|P_{0}\right\|_{\infty}>\lim \sup _{x \rightarrow \infty}\left|P_{0}(x)\right| .
$$

Let also $\lambda$ and $\mu$ be two measures in $M(G)$ such that

$$
\|\lambda+I\|=\|\mu+I\|=1 \text {, and }(\lambda+I) * \mu \subset \delta+I .
$$

Then there exist a complex number $c$ of modulus 1 and a point $x_{0}$ in $G$ such that

$$
\langle P, \mu\rangle=c P\left(x_{0}\right)
$$

for all $P$ in $C \cap I^{\perp}$ with $\|P\|_{\infty} \leqq|\langle P, \mu\rangle|=1$.

Proof. Since $I$ is a left ideal, the space $C \cap I \perp$ is left-translation invariant. Hence, by (a), we may assume that

$$
\left\|P_{0}\right\|_{\infty}=P_{0}(e)=1>\lim _{x \rightarrow \infty} \sup _{x \rightarrow \infty}\left|P_{0}(x)\right|,
$$

where $e$ denotes the identity of $G$. For any given $\varepsilon>0$, take $\lambda^{\prime}$ in $\lambda+I$ so that $\left\|\lambda^{\prime}\right\|<1+\varepsilon$. Putting

$$
Q_{\varepsilon}(y)=(1+\varepsilon)^{-1} \int_{G} P_{0}(x y) d \lambda^{\prime}(x) \quad(y \in G),
$$

it is easy to see that $Q_{\varepsilon}$ is in $N_{\eta}(I)$, where

Further, by (b), we have

$$
\eta=1-\limsup _{x \rightarrow \infty}\left|P_{0}(x)\right| .
$$

$$
\left\langle Q_{\varepsilon}, \mu\right\rangle=(1+\varepsilon)^{-1}\left\langle P_{0}, \lambda^{\prime} * \mu\right\rangle=(1+\varepsilon)^{-1}\left\langle P_{0}, \delta\right\rangle=(1+\varepsilon)^{-1} .
$$

It follows at once that

$$
\sup \left\{|\langle Q, \mu\rangle|: Q \in N_{\eta}(I)\right\}=1=\|\mu+I\| .
$$

This, combined with Proposition, assures that we can find a measure $v$ in $M(G)$ such that

$$
\|\nu\|=1, \quad \text { and } \quad\langle P, \mu\rangle=\langle P, v\rangle \quad\left(P \in C \cap I^{\perp}\right) .
$$

Let us put

$$
B=\left\{P \in C \cap I^{\perp}:\|P\|_{\infty} \leqq|\langle P, \mu\rangle|=1\right\},
$$

and fix any function $Q$ in $B$. It is easy to see from (i') that

$$
|P(x)|=1 \quad(x \in \operatorname{supp}(\nu), P \in B),
$$

and that $P(x) / Q(x)=$ const on $\operatorname{supp}(v)$ for all $P$ in $B$. Fixing any point $x_{0}$ in 
$\operatorname{supp}(v)$, put $c=\langle Q, \mu\rangle \mid Q\left(x_{0}\right)$; then we have

$$
\langle P, \mu\rangle=\langle P, v\rangle=\left(P\left(x_{0}\right) / Q\left(x_{0}\right)\right)\langle Q, v\rangle=c P\left(x_{0}\right) .
$$

This establishes our theorem.

\section{REFERENCES}

1. K. deLeeuw and Y. Katznelson, On certain homomorphisms of quotients of group algebras, Israel J. Math. 2 (1964), 120-126. MR 30 \#5183.

2. E. Hewitt and K. A. Ross, Abstract harmonic analysis. Vol. I. Structure of topological groups. Integration theory, group representations, Die Grundlehren der math. Wissenschaften, Band 115, Springer-Verlag, Berlin and New York, 1963. MR 28 \#158.

3. N. Leblanc, Sur un théorème de deLeeuw et Katznelson, C.R. Acad. Sci. Paris Sér. A-B 269 (1969), A640-A642. MR 40 \#4691.

4. S. Saeki, Homomorphisms of tensor algebras, Tôhoku Math. J. (2) 23 (1971), 173199.

Department of Mathematics, Tokyo Metropolitan University, Fukazawa-Cho, Setagaya, Tokyo, JAPAN

Current address: Department of Mathematics, Kansas State University, Manhattan, Kansas 66502 